stand, lag dies bei 15- bis 19-Jährigen wesentlich höher: unter oralen kombinierten Antikonzeptiva 1,8-fach, unter nur progesteronbasierten Präparaten 2,3-fach. Rein lokal wirkende hormonelle Antikonzeptiva wie Vaginalringe oder intrauterine Systeme führten zu einem noch höheren Risiko, vermutlich gelangt hier vergleichsweise hoch dosiertes Progesteron in die Blutbahn.

\section{Kommentar}

Die Untersuchung liefert auf der Grundlage einer Teilpopulation von dänischen Frauen zwischen 15 und 34 Jahren und damit einer eindrucksvollen empirischen Basis in einem prospektiven Design klare Belege dafür, dass insbesondere bei jüngeren Frauen die Gabe von progesteronhaltigen Antikonzeptiva zu einem erhöhten Risiko für Depression beitragen kann. Dieser Be- fund liefert einen wichtigen klinischen Hinweis: Bei der Verschreibung von progesteronhaltigen Antikonzeptiva ist mit einer erhöhten Depressionswahrscheinlichkeit und mit einem erhöhten Interventionsbedarf zu rechnen.

Prof. Dr. med. Volker Arolt

Skovlund CW et al. Association of Hormonal Contraception With Depression. JAMA Psychiatry $2016 ; 73$ (11): $1154-62$

\title{
Lässt sich das Ansprechen auf EKT vorhersagen?
}

\section{Die Elektrokonvulsionstherapie (EKT) ist eines der wirksamsten Verfahren für die Behandlung von Patienten mit schweren Depressionen. Allerdings sprechen $20-40 \%$ der Patienten nicht darauf an.}

W ährend das Verfahren der EKT technisch relativ einfach ist, erfordert es doch eine gewisse Übung und Praxis sowie die Bereitstellung einer vergleichsweise aufwändigen Infrastruktur, auch wegen der erforderlichen Kurznarkose und Muskelrelaxation. Es wäre sowohl im Hinblick auf die betroffenen $\mathrm{Pa}$ tienten als auch auf die Praktikabilität der EKT von großem Nutzen, wenn Biomarker vorhanden wären, die Aussagen über ein Ansprechen eines individuellen Patienten zulassen würden.

Vergleichsweise wenige Untersuchungen haben sich bisher mit der Vorhersage des Ansprechens auf EKT beschäftigt. Van Waarde et al. [MolPsychiatry 2015; 20: 609-14] untersuchten im funktionellen MRI Resting-States mit einem Mustererkennungsverfahren. Es gelang ihnen, zwei Netzwerke zu identifizieren, die eine individuelle Vorhersage ermöglichten: Ein Netzwerk im dorsomedialen frontalen Kortex mit dem dorsolateralen präfrontalen Kortex, dem orbitofrontalen Kortex und dem posterioren Cingulum zeigte eine Sensitivität von $84 \%$ und eine Spezifität von $85 \%$. Ein weiteres Netzwerk im anterioren Cingulum zeigte eine Sensitivität von $80 \%$ und eine Spezifität von $75 \%$. Diese Ergebnisse waren ermutigend, da zum ersten Mal aufgrund einer statistisch komplexen Analyse von Netzwerkfunktionen des Gehirns eine Vorhersage der individuellen EKT-Response erfolgen konnte. Allerdings ist ein funktionelles
MRT vergleichsweise aufwendig. Redlich und Dannlowski gingen in ihrer Studie einen anderen Weg, der möglicherweise größere Bedeutung für die praktische Anwendung hat, da er lediglich auf der Analyse struktureller MRTAufnahmen basiert. Teilnehmer waren schwer depressive Patienten, die EKT zusätzlich zu Antidepressiva erhielten ( $n=23)$, oder ausschließlich mit Antidepressiva behandelt wurden $(n=23)$ sowie eine Gruppe gesunder Kontrollpersonen $(n=21)$. Beide Patientengruppen unterschieden sich weder in der Depressionsschwere noch im Hinblick auf die Anzahl der depressiven Episoden oder die Dauer der Erkrankung. Die EKTGruppe wies jedoch eine längere Gesamtdauer der Krankenhausbehandlung und eine längere Indexepisode auf. Bei allen Teilnehmern wurden zwei strukturelle MRT angefertigt, eines zu Beginn der Studie und eines nach im Durchschnitt etwa sechseinhalb Wochen, nachdem sich die Symptomatik der Patienten signifikant gebessert hatte (etwa 13 Punkte im HDRS). Die EKT wurde dreimal pro Woche jeweils mit einem Tag Abstand durchgeführt, bis eine deutliche Besserung erkennbar war (im Mittel 14 Sitzungen). Die MRTScans wurden voxelbasiert morphometrisch ausgewertet. Als mathematische Mustererkennungsverfahren wurden eine Support vector machine (LIBSVM) sowie ein Gaussian process classifier (GPC) angewendet. Um das Ansprechen auf EKT vorherzusagen, wurde die Gruppe der Patienten in diesem Studienteil in Responder $(n=13)$ und NichtResponder $(\mathrm{n}=10)$ unterteilt, wobei eine 50 \%ige Reduktion des HDRS-Scores als Kriterium für die Response festgelegt wurde. Die Anwendung beider Mustererkennungsverfahren ergab in beiden Fällen eine hohe Sensitivität bei deutlich geringerer Spezifität. Die Gesamtakkuratheit wies jedoch mit 78,3 (für LIBSVM) beziehungsweise 73,9 (für GPC) recht befriedigende Werte auf. In der Support Vector Regressionsgleichung zeigte sich immerhin eine starke Assoziation $(\mathrm{r}=0,67 ; \mathrm{p} \leq 0,001)$ zwischen der vorhergesagten quantitativen Veränderung im HDRS-Score einerseits und der tatsächlich erreichten Veränderung.

\section{Kommentar}

Aus klinisch-praktischer Sicht ist bedeutsam, dass mithilfe von Mustererkennungsverfahren und einer vergleichsweise einfach herzustellenden MRT-Strukturaufnahme eine individuelle Vorhersagesicherheit von knapp $80 \%$ erreicht werden konnte. Da es sich bei den entsprechenden Mustererkennungsverfahren um lernende Verfahren handelt, ist zu erwarten, dass bei der Untersuchung größerer Patientengruppen - und vor allem bei der Cross-Validierung in verschiedenen Stichproben - Ergebnisse erarbeitet werden können, die eine noch höhere Spezifität und damit auch noch höhere Akkuratheit beziehungsweise Sicherheit für die individuelle Prognose liefern können.

Prof. Dr. med. Volker Arolt

Redlich $R$ et al. Prediction of individual response to electroconvulsive therapy via machine learning on structural magnetic resonance imaging data. JAMA Psychiatry 2016; 73 (6): 1-8 